\title{
Synchronization Through Uncorrelated Noise in Excitatory-Inhibitory Networks
}

\author{
Lucas Rebscher ${ }^{1}$, Klaus Obermayer ${ }^{1}$ and Christoph Metzner ${ }^{1,2 *}$ \\ ${ }^{1}$ Neural Information Processing Group, Technische Universität Berlin, Berlin, Germany, ${ }^{2}$ Biocomputation Group, School of \\ Physics, Engineering and Computer Science, University of Hertfordshire, Hatfield, United Kingdom
}

OPEN ACCESS

Edited by:

Gennady Cymbalyuk

Georgia State University,

United States

Reviewed by:

Leonid L. Rubchinsky,

Indiana University-Purdue University

Indianapolis, United States

Anthony N. Burkitt,

The University of Melbourne, Australia

*Correspondence:

Christoph Metzner cmetzner@ni.tu-berlin.de

Received: 30 November 2021

Accepted: 12 January 2022

Published: 04 February 2022

Citation:

Rebscher L, Obermayer $K$ and Metzner C (2022) Synchronization

Through Uncorrelated Noise in

Excitatory-Inhibitory Networks.

Front. Comput. Neurosci. 16:825865

doi: 10.3389/fncom.2022.825865
Gamma rhythms play a major role in many different processes in the brain, such as attention, working memory, and sensory processing. While typically considered detrimental, counterintuitively noise can sometimes have beneficial effects on communication and information transfer. Recently, Meng and Riecke showed that synchronization of interacting networks of inhibitory neurons in the gamma band (i.e., gamma generated through an ING mechanism) increases while synchronization within these networks decreases when neurons are subject to uncorrelated noise. However, experimental and modeling studies point towardz an important role of the pyramidal-interneuronal network gamma (PING) mechanism in the cortex. Therefore, we investigated the effect of uncorrelated noise on the communication between excitatory-inhibitory networks producing gamma oscillations via a PING mechanism. Our results suggest that, at least in a certain range of noise strengths and natural frequency differences between the regions, synaptic noise can have a supporting role in facilitating inter-regional communication, similar to the ING case for a slightly larger parameter range. Furthermore, the noise-induced synchronization between networks is generated via a different mechanism than when synchronization is mediated by strong synaptic coupling. Noise-induced synchronization is achieved by lowering synchronization within networks which allows the respective other network to impose its own gamma rhythm resulting in synchronization between networks.

Keywords: synchronization, gamma oscillations, PING, noise, communication through coherence

\section{INTRODUCTION}

Synchronous oscillatory activity in high and low frequency ranges has been proposed to underlie coordinated communication between distributed neural systems (Singer, 1999; Buzsáki and Draguhn, 2004; Buzsáki, 2006; Fries, 2009). Especially, gamma rhythms (high-frequency oscillations in the $30-90 \mathrm{~Hz}$ range) have been studied extensively and have been related to perception (Gray et al., 1989), attention (Fries et al., 2001), memory (Tallon-Baudry et al., 1998), consciousness (Melloni et al., 2007), and synaptic plasticity (Wespatat et al., 2004). Furthermore, pathological brain states in neurological and psychiatric disorders, such as Alzheimer's, autism and schizophrenia, have been linked to dysfunctional neural oscillations in the gamma band (Uhlhaas and Singer, 2006, 2010).

Mechanistically, in vitro and in vivo gamma rhythms are mainly produced by two mechanisms termed interneuron network gamma (ING) and pyramidal-interneuron network gamma (PING) 
(Whittington et al., 2000, 2011; Tiesinga and Sejnowski, 2009). The ING mechanism is based on the mutual inhibition of inhibitory neurons, which act as a gate that temporarily suppresses firing until inhibition wears off and the neurons fire in increased synchrony (Buzsáki and Wang, 2012). On the other hand, the PING mechanism is based on the interplay between excitation and inhibition (Buzsáki and Wang, 2012). Firing of excitatory neurons prompts firing of inhibitory neurons which in turn temporarily suppress further firing, ultimately leading to coherent activity in both groups.

Gamma rhythms have been proposed to underlie efficient communication between different brain regions (Engel et al., 2001; Varela et al., 2001; Fries, 2015). For example, the communication-through-coherence (CTC) hypothesis (Fries, $2005,2015)$ posits that synchronization of two brain regions or circuits in the gamma band allows for a more efficient transfer of information between them. Over the last years, this proposal has been supported by considerable experimental evidence, such as gain modulation of both neural and behavioral responses in the gamma band ( $\mathrm{Ni}$ et al., 2016), attentional enhancement of gamma-band synchrony between neural populations (Bosman et al., 2012; Grothe et al., 2012), and covariations in transfer entropy and gamma-band synchrony (Womelsdorf et al., 2007; Besserve et al., 2015). Naturally, neural regions and their communication are subject to various sources of noise and naively one would assume that noise is detrimental to the quality of the signal transfer between the regions and their synchronization. Computational models have for example confirmed that noise can reduce the synchronization of excitatory-inhibitory (EI) networks (Börgers et al., 2005). However, in non-linear biological systems one can also observe a helpful role of noise under certain conditions (Hänggi, 2002; McDonnell and Ward, 2011). For example stochastic resonance, the noise-induced improved response to a weak signal, has been well documented experimentally and theoretically in several neural systems (Gluckman et al., 1996; Plesser and Tanaka, 1997; Ward et al., 2010). Theoretical work has also demonstrated that noise can restore synchrony in neural systems that have been desynchronized by large heterogeneities in the inputs for example McMillen and Kopell (2003) and that colored noise can induce synchronization in neural populations that show a diversity of intrinsic properties (Zhou et al., 2013). Furthermore, noise-induced facilitation of synchrony is a also well documented in theoretical studies of coupled oscillators (see e.g., Zhou et al., 2002; Goldobin and Pikovsky, 2005). So overall, while naively noise might be assumed detrimental to coherent communication, there is a body of experimental and theoretical work demonstrating its ability to facilitate synchrony between populations.

In a recent computational study, Meng and Riecke (2018) demonstrated that noise can synchronize population rhythms generated by individual oscillator networks. They showed that noise induced synchronization despite the noise input between different oscillator networks being uncorrelated. This between-network synchronization emerges as the uncorrelated noise introduces heterogeneity within the networks, thereby weakening intra-network synchronization, and thus allowing for the second network to control a substantial fraction of the network activity. While they demonstrate that this type of synchronization emerges in different settings, their findings remain restricted to networks coupled by inhibition, i.e., they do not investigate the synchronization of PING networks.

Therefore, in this study, we model two interconnected excitatory-inhibitory networks, producing gamma oscillations through a PING mechanism, in different network settings and analyze how synchronization within and across the networks changes depending on the strength of uncorrelated noise input to the networks. Our results extend the findings from Meng and Riecke (2018) and suggest that uncorrelated noise can also have a supporting role in facilitating inter-regional communication in PING networks. Importantly, our models can be used as a basis to investigate mechanistic explanations for altered neuronal dynamics in psychiatric disorders, since for example, disturbances in neuronal oscillations in the gamma band, especially reduced synchronization, are a key finding in schizophrenia (Uhlhaas and Singer, 2010).

\section{METHODS}

We first replicated the results of Meng and Riecke (2018) using two interacting inhibitory networks producing gamma rhythms via an ING mechanism where each neuron was subject to independent noise. Once we were able to replicate their results in our model implementation, we proceeded to the next step and extended the model to two interacting excitatory-inhibitory networks showing a PING mechanism. In this case, we looked at two variants: the simpler case of all-to-all connectivity and the biologically more plausible case of sparse random connectivity. Conclusively, in the following we define the three scenarios that we simulated, evaluated and compared:

- Scenario 1: In this scenario, we replicated the case of two interacting inhibitory networks with all-to-all connectivity investigated by Meng and Riecke (2018). Importantly, each neuron was subject to uncorrelated noise and both networks displayed rhythmic gamma band activity produced by the ING mechanism.

- Scenario 2: This scenario extended scenario 1 to two all-toall coupled excitatory-inhibitory networks driven by the PING mechanism. It also built the foundation for scenario 3 and potentially more complex variants used in future work.

- Scenario 3: In the last scenario, we moved from all-toall connectivity to random sparse connectivity to assess to which degree independent noise would also be able to have a beneficial effect in networks with a biologically more plausible connectivity.

\subsection{Network Models}

In scenario 1, we considered a model of two coupled inhibitory networks analogous to Meng and Riecke (2018). Both populations consisted of 1,000 inhibitory neurons and received input in form of random uncorrelated spike trains of 800 excitatory neurons according to a Poisson schedule. Each population had recurrent connections and both networks were 


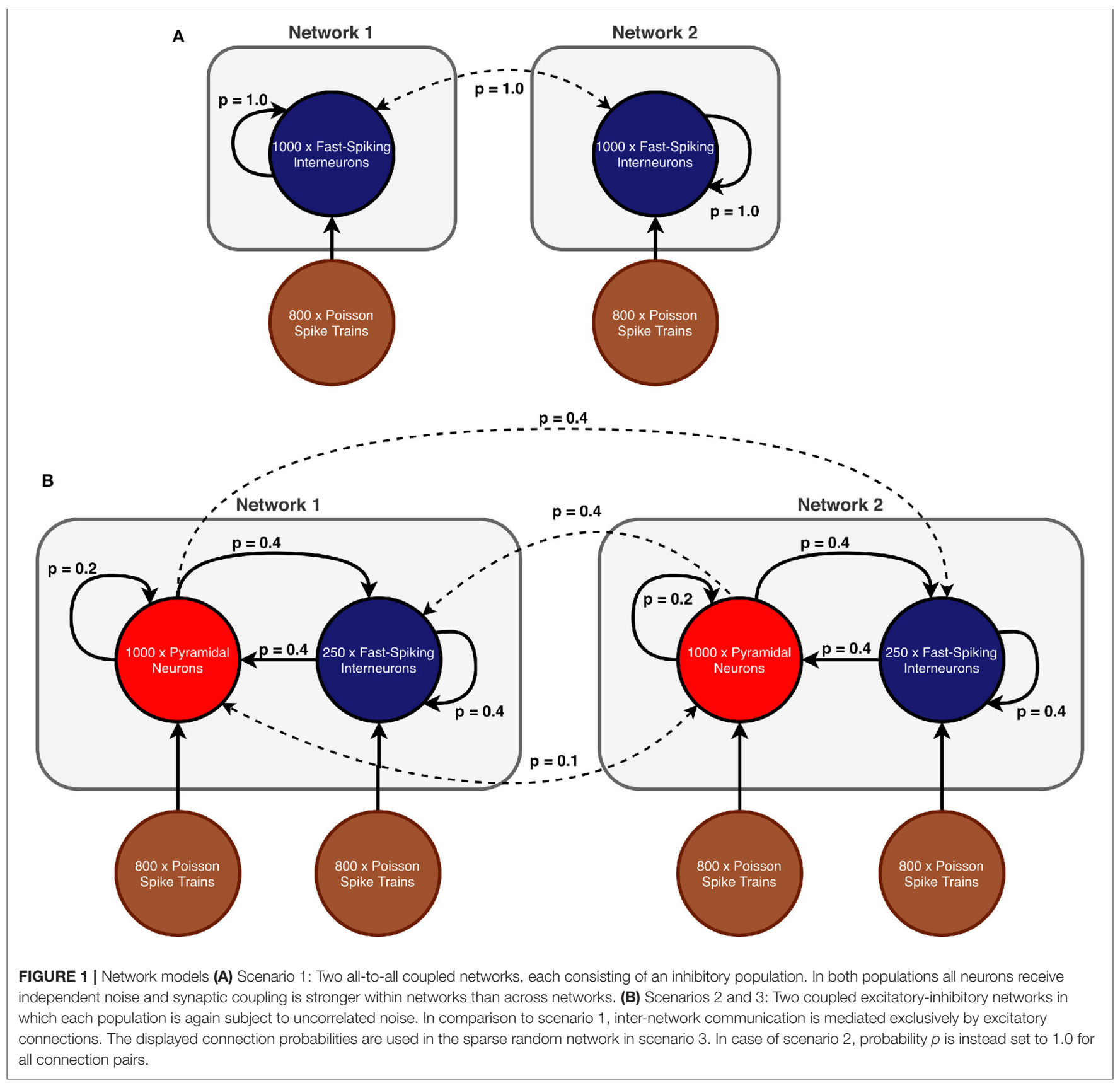

bidirectionally coupled (see Figure 1A for a schematic of the ING network setup). As connectivity in scenario 1 is all-to-all, the connection probability $p$ was 1.0 for all connection types.

In scenarios 2 and 3 , each network consisted of a population of 250 inhibitory neurons as well as a population of 1,000 excitatory neurons (see Figure 1B for a schematic of the setup). The difference between the two scenarios lies in the particular connectivity scheme (all-to-all vs. sparse random) and the synaptic coupling strengths. Excitatory and inhibitory populations within each network were recurrently connected. However, inter-network communication was mediated solely by excitatory synapses that target both the inhibitory and excitatory populations in the respective other network. We explicitly omitted long-range inhibitory connections because connectivity between brain regions (such as V1 and V2 for example) is predominantly excitatory [e.g., in monkey (Tomioka and Rockland, 2007) and other species (McDonald and Burkhalter, 1993; Gonchar et al., 1995; Fabri and Manzoni, 1996, 2004)] and we did not model communication between two populations within a region, where long-range inhibition might be more prevalent.

The connection probabilities were configured according to the specific scenario and are shown in Table 1. While all populations were again subject to uncorrelated noise, the 
TABLE 1 | A list of all relevant model parameters, including parameters of AdEx model, GABA-, and AMPA-mediated synapses, synaptic noise, and connection probabilities.

\begin{tabular}{|c|c|c|}
\hline Parameter & Value & Description \\
\hline$N_{E}$ & 1,000 & Number of excitatory (E) cells \\
\hline$N_{1}$ & 250 & Number of inhibitory (I) cells \\
\hline C & $200 \mathrm{pF}$ & Membrane capacitance \\
\hline$g_{L}$ & $10 \mathrm{nS}$ & Membrane leak conductance \\
\hline$E_{L}$ & $-65.0 \mathrm{mV}$ & Membrane leak reversal potential \\
\hline$E_{W}$ & $-80.0 \mathrm{mV}$ & Adaptation reversal potential \\
\hline$V_{T}$ & $-50.0 \mathrm{mV}$ & Membrane threshold \\
\hline$\Delta_{T}$ & $1.5 \mathrm{mV}$ & Threshold slope factor \\
\hline$V_{r}$ & $-70.0 \mathrm{mV}$ & Reset voltage \\
\hline$\tau_{\text {ref }}$ & $1.0 \mathrm{~ms}$ & Length of refractory period \\
\hline$a_{e x c}$ & $4.0 \mathrm{nS}$ & Subthreshold adaptation parameter of E cell \\
\hline$b_{\text {exc }}$ & $40.0 \mathrm{pA}$ & Spike-frequency adaptation parameter of E cell \\
\hline$a_{\text {inh }}$ & $0 \mathrm{nS}$ & Subthreshold adaptation parameter of I cell \\
\hline$b_{\text {inh }}$ & $0 \mathrm{pA}$ & Spike-frequency adaptation parameter of I cell \\
\hline$\tau_{A M P A}$ & $3.0 \mathrm{~ms}$ & AMPA decay time Constant \\
\hline$E_{A M P A}$ & $0.0 \mathrm{mV}$ & AMPA reversal potential \\
\hline$\tau_{G A B A}$ & $6.0 \mathrm{~ms}$ & GABA decay time Constant \\
\hline$E_{G A B A}$ & $-70.0 \mathrm{mV}$ & GABA reversal potential \\
\hline$J_{\text {etoe }}$ & $0.01 \mathrm{nS}$ & Synaptic coupling strength - E $\rightarrow E$ within network \\
\hline$J_{\text {etoi }}$ & $0.05 \mathrm{nS}$ & Synaptic coupling strength - E $\rightarrow$ I within network \\
\hline$J_{\text {itoi }}$ & $0.7 \mathrm{nS}$ & Synaptic coupling strength - I $\rightarrow$ I within network \\
\hline$J_{\text {itoe }}$ & $0.5 \mathrm{nS}$ & Synaptic coupling strength - I $\rightarrow$ E within network \\
\hline$J_{\text {ppee }}$ & $0.01 \mathrm{nS}$ & Synaptic coupling strength $-E \rightarrow E$ across networks \\
\hline$J_{\text {ppei }}$ & $0.03 \mathrm{nS}$ & Synaptic coupling strength - E $\rightarrow$ I across networks \\
\hline$J_{\text {ppii }}$ & $0.0 \mathrm{nS}$ & Synaptic coupling strength $-\mathrm{I} \rightarrow$ I across networks \\
\hline petoe & 0.2 & Connection probability from $\mathrm{E}$ to $\mathrm{E}$ \\
\hline$p_{\text {etoi }}$ & 0.4 & Connection probability from $\mathrm{E}$ to I \\
\hline$p_{\text {itoe }}$ & 0.4 & Connection probability from I to $E$ \\
\hline$p_{\text {itoi }}$ & 0.4 & Connection probability from I to I \\
\hline pppee & 0.1 & Inter-network connection probability from $E$ to $E$ \\
\hline$p_{\text {ppei }}$ & 0.4 & Inter-network connection probability from E to I \\
\hline$\delta_{\text {net }}$ & $0.0 \mathrm{~ms}$ & Inter-network communication delay \\
\hline$N_{p}$ & 800 & Number of neurons in a Poisson group \\
\hline$\mu_{\text {ext }}$ & $300 \mathrm{~Hz}$ & Mean external noise input \\
\hline$\sigma^{2}$ & $1.0 \mathrm{~Hz}$ & Noise strength \\
\hline$p$ & 0.75 & Poisson rate ratio \\
\hline
\end{tabular}

Parameters such as coupling strengths and connection probabilities vary across scenarios.

inhibitory populations received a lower proportion of noise to avoid that the spiking of inhibitory neurons became dominated by the noise input, since this would have caused the PING rhythms to collapse (Börgers and Kopell, 2003).

\subsection{Neuron Model}

As a neuron model we used the adaptive exponential integrateand-fire (AdEx) model proposed by Brette and Gerstner (2005) and its membrane potential time course is described in Equation (1). To improve readability, we extracted the internal membrane dynamics to the current $I_{\text {ion }}$ in Equation (2).
Adaptation is modeled by the parameter $w$ as defined in Equation (3).

$$
\begin{aligned}
C \frac{d V}{d t} & =I_{\text {ion }}+I_{A M P A}+I_{G A B A}+I_{\text {ext }} \\
I_{\text {ion }}(V) & =-g_{L}\left(V-E_{L}\right)+g_{L} \Delta_{T} \exp \left(\frac{V-V_{T}}{\Delta_{T}}\right)-w \\
\tau_{w} \frac{d w}{d t} & =a\left(V-E_{w}\right)-w
\end{aligned}
$$

where $C$ is the membrane capacitance, $E_{L}$ the leak reversal potential, $E_{w}$ the adaptation reversal potential, $V$ the membrane voltage at time step $t, V_{T}$ the membrane threshold, $V_{\text {reset }}$ the reset potential, $\Delta T$ the slope factor, $a$ the adaptation coupling parameter, $\tau_{w}$ the adaptation time constant, and $g_{L}$ the leak conductance. When the membrane potential exceeds the spiking threshold, the voltage is reset to $V_{r}$ and clamped for a refractory time $T_{\text {ref }}$. Furthermore, the spike-triggered adaptation increment $b$ is added to the adaptation current. Each neuron received postsynaptic currents $I_{G A B A}$ and $I_{A M P A}$ which we further specify below. Finally, the current $I_{\text {ext }}$ represents the external noise input that we define below.

\subsection{Synapse Model}

Synaptic currents for GABAergic as well as glutamatergic (AMPA) synapses are defined in Equations (4) and (6), respectively. We used a conductance-based model and the respective synaptic conductance is modeled as a dynamic variable with an instantaneous rise on each pre-synaptic spike and an exponential decay over time. The exponential decay is defined in Equations (5) and (7) for AMPA and GABA, respectively.

$$
\begin{aligned}
I_{A M P A} & =g_{A M P A}\left(E_{A M P A}-V\right) \\
\frac{d g_{A M P A}}{d t} & =-\frac{g_{A M P A}}{\tau_{A M P A}} \\
I_{G A B A} & =g_{G A B A}\left(E_{G A B A}-V\right) \\
\frac{d g_{G A B A}}{d t} & =-\frac{g_{G A B A}}{\tau_{G A B A}}
\end{aligned}
$$

where $g_{G A B A}$ and $g_{A M P A}$ are the dynamic synaptic conductances, $E_{A M P A}$ and $E_{G A B A}$ the characteristic reversal potentials, $V$ the membrane potential of the post-synaptic neuron, and lastly $\tau_{A M P A}$ and $\tau_{G A B A}$ represent the time constants that define the speed of the exponential decay.

The synaptic conductances $g_{G A B A}$ and $g_{A M P A}$ is modeled for each neuron and their instantaneous rise is described by the following update rules executed on every pre-synaptic spike:

$$
\begin{array}{rlrl}
g_{A M P A_{I}}+ & =J_{\text {etoi }} & g_{A M P A_{E}}+ & =J_{\text {etoe }} \\
g_{A M P A_{P} E}+ & =J_{\text {ppee }} & g_{A M P A_{P} I}+=J_{p p e i} \\
g_{G A B A_{E}}+=J_{\text {itoe }} & g_{G A B A_{I}}+=J_{i t o i} \\
g_{G A B A_{P} I}+=J_{\text {ppii }} & &
\end{array}
$$

where $J_{\text {etoi }}, J_{\text {etoe }}, J_{\text {itoe }}$, and $J_{\text {itoi }}$ are the synaptic coupling strengths between excitatory or inhibitory neurons within a network, 
whereas $J_{p p e e}, J_{p p e i}$, and $J_{p p i i}$ are the coupling strengths across networks. We deliberately left out the coupling strength $J_{p p i e}$ of synapses originating from inhibitory neurons in one network and targeting excitatory neurons in another network, since this type of connection is not present in any of our scenarios. Further, note that $J_{p p i i}$ was only present in the inhibitory networks of scenario 1 whereas $J_{\text {ppee }}, J_{\text {ppei }}$ were only present in the excitatory-inhibitory networks of scenarios 2 and 3 .

\subsection{Noise Model}

We adopted the noise framework that Meng and Riecke (2018) used to generate uncorrelated noise for each neuron. Each population in every scenario was subject to substantial synaptic noise in form of a group of $N_{p}=800$ Poisson neurons. We used the mean input $\mu_{v}$, the noise strength $\sigma_{v}{ }^{2}$ and the Poisson rate ratio $p$ as free parameters in our explorations. The mean input $\mu_{v}$ describes the mean effect on the membrane potential caused by the noise and the noise strength is simply its variance.

For each Poisson neuron we generated spike trains according to a Poisson process with a rate $\lambda$ that was dependent on the free parameters $\mu_{v}$ and $\sigma_{v}{ }^{2}$, as shown in Equation (12), where $N_{p}$ (e.g., $N_{p}=800$ ) is the number of Poisson neurons.

$$
\lambda=\frac{\mu_{v}^{2}}{\sigma_{v}^{2} * N_{p}}
$$

Importantly, the rate differed between the two networks and network 2 received a lower rate than network 1 . This ultimately determined the natural frequency of the network activity and was performed to set the networks to a desynchronized state. Therefore, the difference in the noise input between the two networks lay only in the rate $\lambda$, the strength of a Poisson generated spike stayed the same within and across networks. The rate difference between network 1 and network 2 was controlled by the Poisson rate ratio $p$ with $p \in[0,1]$ as follows

$$
\lambda_{2}=p \lambda_{1}
$$

Besides generating spike trains, we also needed to model the impact of a generated pre-synaptic spike on a post-synaptic neuron. This was modeled in form of an external current $I_{\text {ext }}$ with

$$
I_{\text {ext }}(t)=g_{P}\left(V_{T}-V_{\text {reset }}\right) \Delta v P(t)
$$

The conductance $g_{P}$ was included to ensure correct physical units and we set it to a constant value of $1 n S . P(t)$ is the number of pre-synaptic Poisson spikes arriving at time step $t$ at the respective post-synaptic neuron. The difference between the membrane threshold $V_{T}$ and the reset potential $V_{\text {reset }}$ of the respective post-synaptic neuron is precisely the amount of voltage needed to produce a spike independent of its current membrane potential. However, the extent of this driving force is controlled by a dimensionless input strength $\Delta v$. Analog to the rate $\lambda, \Delta v$ is also dependent on the free parameters $\sigma^{2}$ and $\mu$ according to

$$
\Delta v=\frac{\sigma^{2}}{\mu}
$$

We can observe based on Equations (12) and (15) that an increase in the noise strength $\sigma^{2}$ leads on one hand to an increase in the spike strength $\Delta v_{i}$ and on the other hand to a decrease in the rate $\lambda$.

Note that, as Meng and Riecke did in their original model, we here also used mean input $\mu$ and noise strength $\sigma_{v}^{2}$ as free parameters instead of the Poisson spike rate $\lambda$ and the input strength $\Delta v$. This ensures that the noise strength, which is characterized by $\sigma^{2}$, is the same for all neurons across all networks. As shown in Meng and Riecke (2018), the mean input can be recovered via the spiking rate and the input strength through $\mu_{v}=\lambda \cdot \Delta v$ and the noise strength through $\sigma_{v}^{2}=$ $\lambda \cdot(\Delta v)^{2}$

\subsection{Parameter Explorations}

Two-dimensional explorations were visualized in form of heat maps where the colors were mapped to the value of a specific measure. We decided to use three different measures which are presented in the following.

\subsubsection{Phase Synchronization Between Two Networks}

The synchronization between the networks is perfect if the phase difference over time is constant as we might have considerable delay regulated by parameter $\delta_{\text {net }}$. Thus, the synchronization between networks was quantified by the mean phase coherence of the networks' activity. We used the average $n_{i}$ of the neurons' voltage traces inside a network as a surrogate for the network level activity [as for example measured as an local field potential (LFP)]

$$
n_{i}=\frac{1}{N} \sum_{k=1}^{N} v_{k}
$$

where the sum is over all $N$ neurons inside network $i$ and $v_{k}$ is the recorded voltage trace of neuron $k$. While we acknowledge that LFP proxies based synaptic currents of excitatory cells usually more closely match experimentally recorded LFPs (Mazzoni et al., 2008, 2015; Barbieri et al., 2014), we used the average membrane potential in our work for two reasons: (1) Meng and Riecke also used it in their work (Meng and Riecke, 2018) and thus our work can be directly compared to theirs and (2) defining a current-based measure for the inhibitory networks of scenario 1 that closely matches experimentally measured LFPs is not straightforward, and, therefore, our choice of LFP avoids using different measures for the ING and PING networks and makes the results for the different network settings comparable. For scenario 3 (EI network), we repeated our analysis with an LFP proxy based on the sum of the absolute values of AMPA and GABA currents, which captures approximately $90 \%$ of the variance of experimentally measured LFPs (Mazzoni et al., 2015), and did not find any differences.

Before extracting the phase information, we bandpass filtered the signal $n_{i}$ to ensure that the extracted phase information is meaningful. As a filter we used a secondorder Butterworth filter with a lowcut frequency of $30 \mathrm{~Hz}$ and a highcut frequency of $120 \mathrm{~Hz}$ implemented in the scipy ${ }^{1}$

${ }^{1}$ https://www.scipy.org/ 
package scipy.signal.filter_design.butter. Next, we extracted the phases $\varphi_{X}$ from the filtered signal of population $X$ by applying the Hilbert transform implemented in the scipy package under scipy.signal.hilbert. Finally, the mean phase coherence between the two networks A and B was calculated as

$$
R=\left|\frac{1}{T} \sum_{t=1}^{T} e^{i\left(\varphi_{A}(t)-\varphi_{B}(t)\right)}\right|
$$

where $\varphi_{X}(t)$ is the phase of the signal of population $X$ at time $t$ and $T$ the total number of time steps. If the phase difference between two signals is constant over time $R=1$. In this case, the signals are said to be phase locked. On the other hand, a low $R$ near 0 typically also means a low correlation between the phases (although one can artificially create signals with non-zero correlation that have an $R=0$ ) and if the two signals have no correlation then $R=0$.

\subsubsection{Phase Synchronization Within a Population}

When determining the phase synchronization within a population, we looked at the signals of all individual neurons in one population and, accordingly, calculated the synchronization of these signals. Further, the same preprocessing steps as above were applied. However, instead of using the mean phase coherence, we chose the Kuramoto order parameter because perfect synchronization inside a homogeneous population is expected to be equivalent to zero phase lag synchronization. The Kuramoto order parameter was then calculated according to

$$
K=\left|\frac{1}{T} \sum_{t=1}^{T} \frac{1}{N} \sum_{j=1}^{N} e^{i \phi_{j}(t)}\right|
$$

where $\phi_{j}(t)$ is now the phase of signal of an individual neuron $j$ at time $t, T$ the total number of time steps and $N$ the total number of neurons in the population.

The reason for using two different synchronization measures here, is that on the one hand we wanted to measure synchronization within a network, where we assume the neurons of the network to display zero phase lag activity if the network is fully synchronized and on the other hand, we wanted to explore the synchrony between two networks, where full synchrony would also entail the scenario where the two networks have a constant non-zero phase lag (e.g., introduced by the axonal delay of the connection between them). Therefore, we chose the Kuramoto order parameter for the within network synchronization, because it is sensitive to the phase lag, and the mean phase coherence for the between network synchronization, because it ignores non-zero phase lags as long as they are onstant.

\subsubsection{Frequency Locking of Two Networks}

The difference in the frequencies of two oscillators is a measure of frequency locking (also sometimes referred to as entrainment) (Pikovsky et al., 2003). To quantify frequency locking between two networks, we used the same measure as Meng and Riecke (2018), namely the ratio between the dominant frequencies of the networks. First, the network signals were transformed to the frequency-domain by applying Welch's method (Welch, 1967) offered by the Python package matplotlib ${ }^{2}$ in the module matplotlib.mlab.psd. Next, the dominant frequency $f_{i}$ of each of the two networks was determined by extracting the frequency with the highest power. Finally, the ratio between the two extracted frequencies was calculated as follows

$$
r=\frac{f_{2}}{f_{1}}
$$

with $f_{2} \leq f_{1}$ and $f_{1}, f_{2}>0$. The larger dominant frequency corresponds to $f_{1}$ and the lower one to $f_{2}$ to ensure that $r \in(0,1]$. A ratio of 1 indicates perfect frequency locking as the two frequencies equal whereas values close to 0 indicate maximal mismatch between the two frequencies. Noteworthy, in our simulations, network 1 always had a greater or equal dominant frequency than network 2 because it received stronger noise input.

\subsection{Code and Simulations}

The source code is publicly accessible on GitHub ${ }^{3}$. All code was written in Python 3.7. We used the spiking neural simulator Brian 2 (Stimberg et al., 2019) (2.3.0.2) for model simulations. To run large scale parameter explorations we used the exploration library mopet (Cakan and Rebscher, 2020) (0.1.3). All plots were created with the Python library matplotlib ${ }^{4}$ (3.1.2) and signal processing steps were performed with the scipy (see text footnote 1) (1.4.1) package.

To ensure that our simulation results were robust and could be reproduced in a different simulation setting, we evaluated our results with different step sizes $(1.0 \mathrm{~ms}, 0.5 \mathrm{~ms}$, $0.05 \mathrm{~ms})$, simulation durations $(0.5 \mathrm{~s}, 1 \mathrm{~s}, 3 \mathrm{~s}, 5 \mathrm{~s})$ and integration methods. Regarding the integration methods, we used three different methods implemented in Brian $2^{5}$ : heun (stochastic Heun method), euler (forward Euler integration), and milstein (derivative-free Milstein method). The results did not vary noticeably in all cases. For our final simulations, we used a step size of 0.05 , a duration of $5 s$, and the heun integration method.

\section{RESULTS}

\subsection{Uncorrelated Noise Can Synchronize Networks of ING Populations}

In scenario 1, we first investigated the effect of uncorrelated noise input on the synchronization of two coupled inhibitory networks that produce a gamma oscillation via an ING mechanism (similar to Meng and Riecke, 2018).

In this scenario, both inhibitory networks were driven by external, uncorrelated noise. Importantly, network 1 had a higher natural frequency than network 2 as network 1 was subject to stronger noise. This difference was controlled by the Poisson rate ratio parameter $p$. We then explored whether, and if so to what extent, certain noise strengths $\sigma^{2}$ might be able to enhance the

\footnotetext{
${ }^{2}$ https://matplotlib.org/

${ }^{3}$ https://github.com/ChristophMetzner/Synchronization-by-Uncorrelated-Noise

${ }^{4}$ https://pypi.org/project/matplotlib/

${ }^{5}$ https://brian2.readthedocs.io/en/stable/user/numerical_integration.html
} 
synchronization between the networks in relation to the Poisson rate ratio $p$.

\subsubsection{Exploration}

For a fixed Poisson rate ratio $p$, e.g., $p=0.85$, we observed that the dominant frequency ratio and the phase synchronization between networks improved if the noise strength $\sigma^{2}$ was sufficiently increased (Figure $\mathbf{2 A}$ ). At the same time, the within phase synchronization of the respective inhibitory networks decreased. For sufficiently large Poisson rate ratios $p$ (typically $p>0.75)$, an increase above a certain threshold in the noise strength generally improved the synchronization between the networks initially (Figure 2A). Additionally, higher noise Poisson rate ratios seemed to lower the minimal noise strength $\sigma^{2}$ required to transition the two networks to a frequency and phase locked state.

Furthermore, the results of the one-dimensional exploration (Figure 2B) highlighted the opposing relationship between within and across network synchronization when noise strength $\sigma^{2}$ was increased. In this case, the Poisson rate ratio was initially set to $p=0.85$.

Again, the dominant frequency ratio and the mean phase coherence between the networks increased while synchronization within the networks decreased. Interestingly, once $\sigma^{2}$ reached 1.4, the network abruptly transitioned to a frequency locked state as the dominant frequency ratio jumped to and stayed at 1.0 while phase locking was increased only slowly with further increase in strength. Although an increase in noise could apparently improve inter-network synchronization, the beneficial effect was limited. In this case, the maximum was reached at $\sigma^{2}$ of 1.9 and a further increase worsened both within and across network synchronization. Additionally, the dominant frequency ratio began to fluctuate at a noise strength of 3.0.

\subsubsection{Analysis of Three Distinct States}

We then proceeded to compare three parameter configurations, each of which showed a different state in the scenario of two coupled inhibitory networks:

State 1: Unsynchronized activity across and synchronized activity within networks-Weak noise and weak internetwork coupling (Figure 3A)

State 2: Synchronized activity across and within networks-Weak noise and strong inter-network coupling (Figure 3B)

State 3: Synchronized activity across and unsynchronized activity within networks-Strong noise and weak inter-network coupling (Figure 3C). In contrast to the second state, synchronization within the networks was impaired, pointing towardz a different synchronization mechanism.

Additionally to the case of weak inter-network coupling with weak and strong noise (state 1 and 3, respectively), which was already explored in Meng and Riecke (2018), we here also included the case of strong inter-network coupling with weak noise. As we will demonstrate later, state 2 and 3 both displayed synchronization across networks, however, the underlying synchronization mechanism was fundamentally different. Thus, we here extend the findings of Meng and Riecke (2018) for ING networks.

State 1 (Figure 3A) was characterized by a high withinnetwork synchronization and no synchronization across networks. This was not surprising, since the coupling strength between networks and the noise strength were both comparatively weak so that the behavior of one network was not considerably influenced by activity from the respective other network. Specifically, there was a mismatch between the dominant frequencies of the two networks ( $55 \mathrm{~Hz}$ for network 1 and $39 \mathrm{~Hz}$ for network 2), because network 2 received noisy spike input with a lower rate determined by the Poisson rate ratio $p$, in this case $p=0.85$. Unsurprisingly, the temporal evolution of the networks' phases also did not display any coherence between the two networks. Further, the coherent and rhythmic firing of the inhibitory neurons confirmed the high phase synchronization within each network.

For state 2 (Figure 3B), coupling strength between the networks was increased until the two networks synchronized. This state was characterized by a homogeneous behavior within networks and across networks, demonstrated by the overlapping power spectra and matching dominant frequencies, confirming a 1:1 frequency locking. This was further confirmed by the evolution of the phases of the networks. Interestingly, while both networks again displayed rhythmic behavior, the participation of neurons in network 2 was markedly reduced compared to network 1 and only a small fraction of the neurons participated in each cycle. The networks synchronized through a winner-takes-all effect in favor of the faster network 1. By activating inhibitory neurons in network 2, network 1 suppressed all neurons in network 2 that did not spike precisely in cycles of the inhibitory rhythm of network 1 . Over time, the population activity of network 2 synchronized with the population activity of network 1. Again, the total power in network 2 was lower compared to network 1, since network 2 received a lower proportion of the noise input and was subject to strong inhibition from the faster network 1 .

In state 3 (Figure 3C), when we increased noise strength while keeping inter-network coupling weak, the networks also transitioned to a 1:1 phase and frequency locked state. But contrary to state 2, this was achieved through the increased variability in the membrane potentials, which weakened the synchronization within a network, while allowing a variable fraction of neurons in the slower network 2 to participate in cycles of network 1 . The increase in noise strength increased spike time variability inside a network, thereby weakening the gating effect of inhibition, which led to an acceleration of population rhythms and explained the higher dominant frequencies in the power spectra of both networks compared to state 1 and 2. Similar to state 2 , the dominant frequencies matched, but the noise led to a spread in the power of both network signals, especially in network 2. Furthermore, the networks were in general 1:1 phase locked despite some occasional irregularities caused by the higher variability of spiking in both networks due to the strong noise. In state 2, strong inhibition 

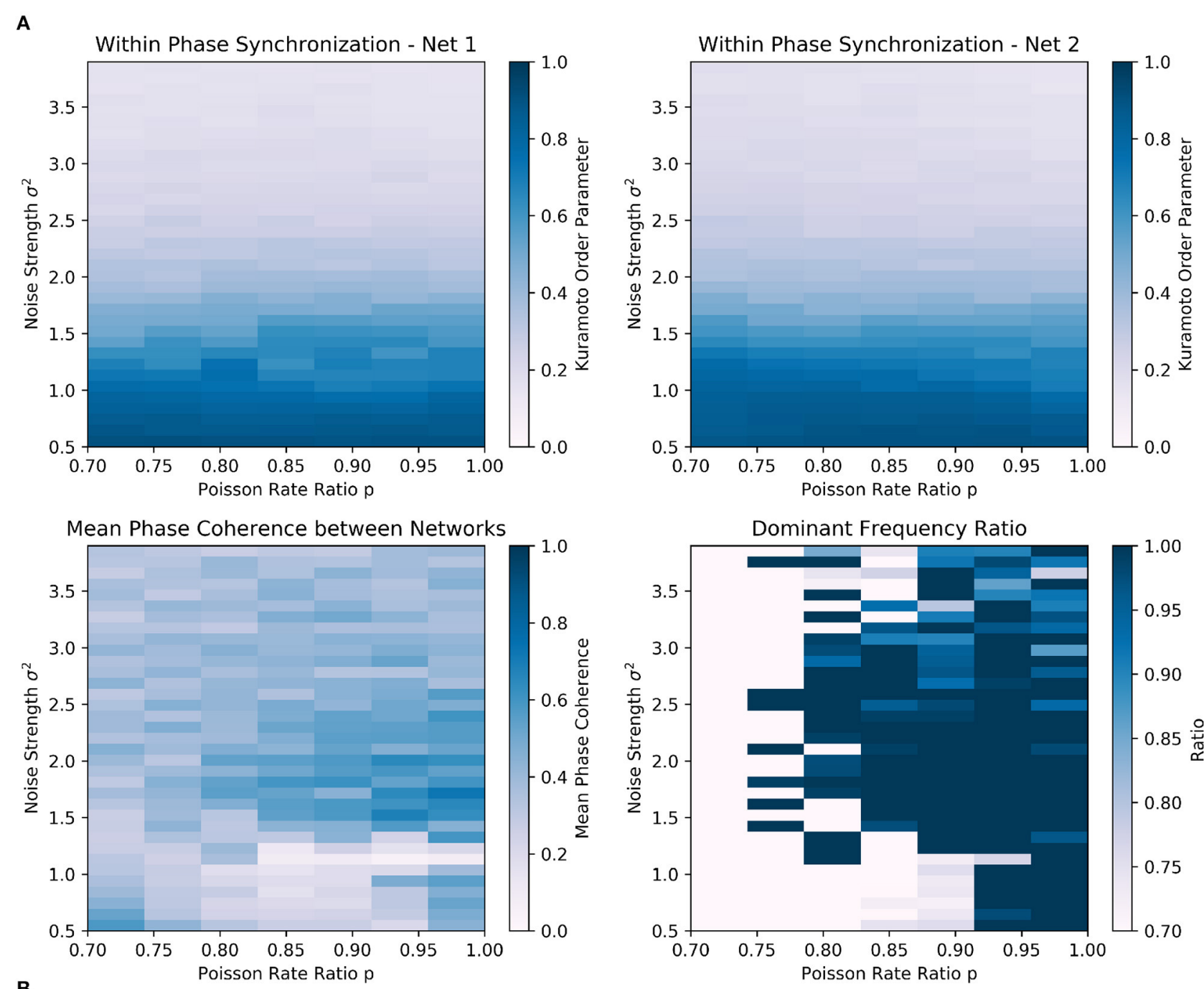

B

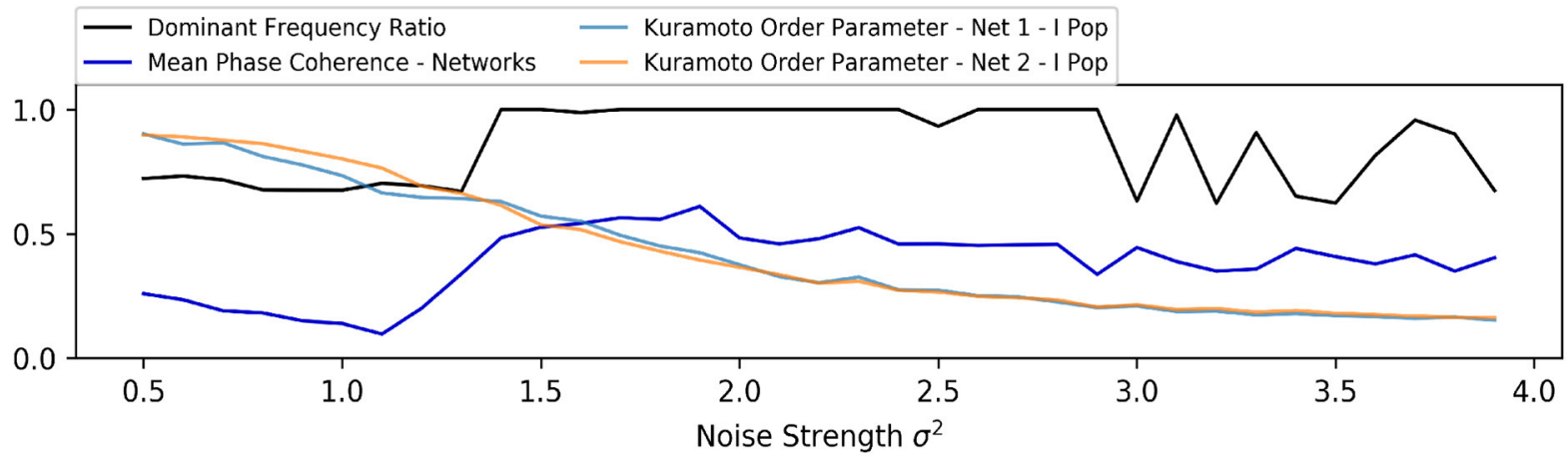

FIGURE 2 | Scenario 1-Exploration of two interacting all-to-all connected inhibitory networks driven by the ING mechanism. (A) Heat maps visualizing two-dimensional exploration over noise strength $\sigma^{2}$ and Poisson rate ratio $p$. The heat maps in the first row encode the phase synchronization within the respective inhibitory network while the bottom row displays phase synchronization and dominant frequency ratio across the two networks. (B) Visualization of a one-dimensional exploration over noise strength $\sigma^{2}$ values from 0.5 to 4.0 in 0.1 steps. The Poisson rate ratio of noise was set to $p=0.85$.

from network 1 led to suppression of spikes in network 2 if they did not fall into cycles of network 1, explaining their synchronization over time. However, in state 3 synchronization was induced by a different mechanism. Specifically, the strong noise weakened the within-network synchronization (especially in the inhibitory population) as inhibitory feedback was not 


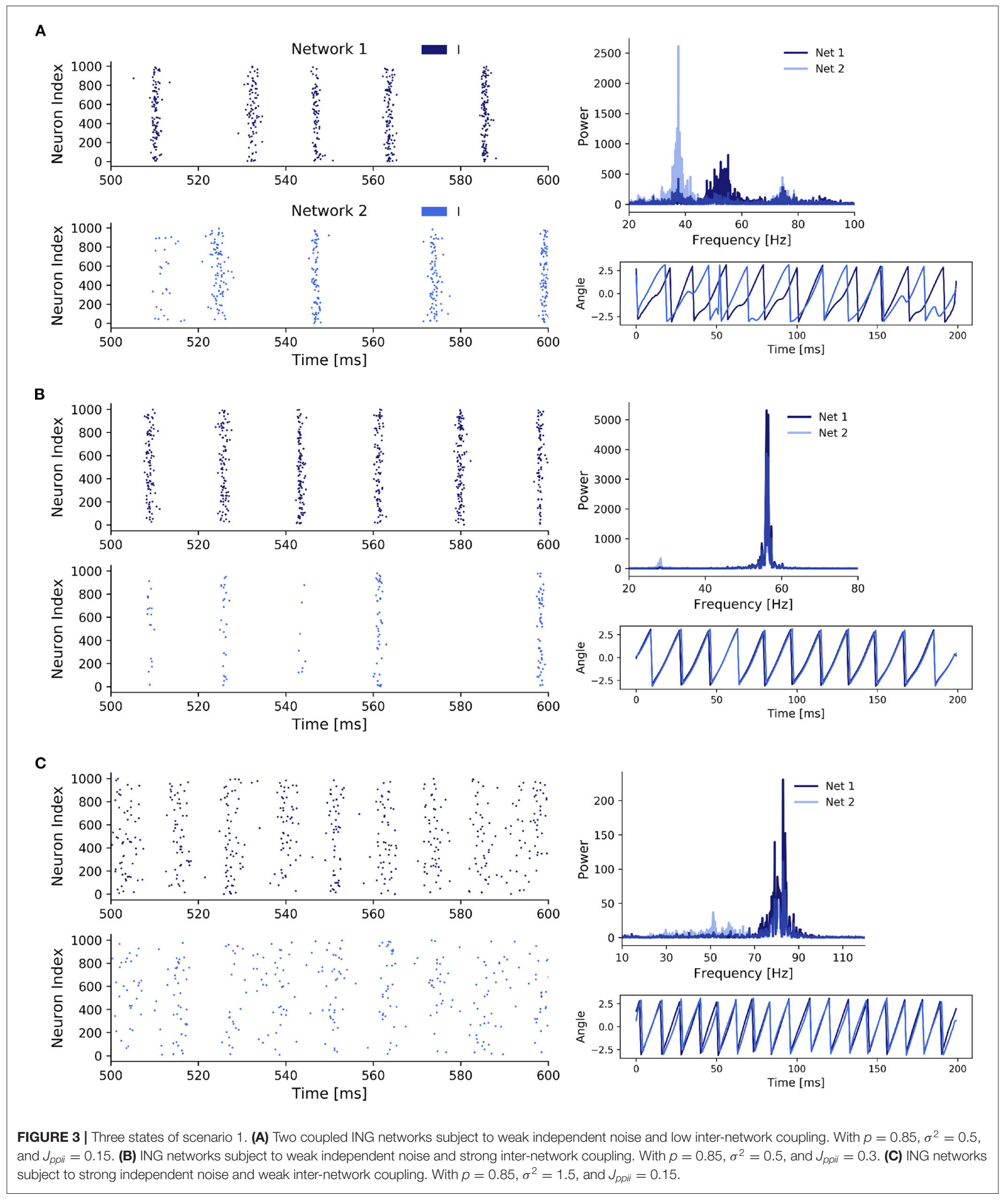

able to completely cancel out the noise-induced randomness contrary to the weak noise in state 2 . This reduced inhibition inside a network enabled spikes of neurons in network 1 to have a bigger influence on the neurons in network 2, explaining the sparse spike participation of network 2 in cycles of network 1. 


\subsection{Uncorrelated Noise Can Synchronize Networks of PING Populations}

Next, we explored the effects of uncorrelated noise in interconnected networks of excitatory and inhibitory populations. We investigated two types of EI networks, allto-all coupled (scenario 2) and random, sparsely coupled networks (scenario 3). However, since the findings were independent of the particular coupling type we only present the results for the biologically more plausible scenario of sparse and random connectivity between neurons in each population. The results for the all-to-all coupled networks can be found in the Supplementary Figure S1.

\subsubsection{Exploration}

In the case of two random sparsely connected excitatoryinhibitory networks, increased noise strength could indeed synchronize the two networks with respect to phase and frequency locking (Figure 4A). Similar to scenario 1, for a fixed Poisson rate ratio $p$, increased noise strength $\sigma^{2}$ eventually pushed the unsynchronized networks to a synchronized state. Furthermore, there was again an inverse relationship between the Poisson rate ratio $p$ between the networks and the noise strength required for the networks to synchronize their rhythms.

Interestingly, the PING rhythms seemed to be more robust against noise than the ING rhythms in scenario 1. It required comparatively higher noise strengths to produce any noticeable changes in the behavior of each network. However, as soon as the noise strength was increased sufficiently, high values of the mean phase coherence and the dominant frequency ratio between the two networks could be observed. Again, this beneficial effect was bounded from above and further increases in noise strengths led to a deterioration of both within and across network synchronization, suggesting that network behavior was mainly determined by the external noise input in this parameter regime.

The parameter space in which frequency and phase locking could be observed was reduced and limited to input Poisson rate ratios above 0.80 (Figure 4A). The sensitivity to noise was also visible based on the fast decline of within phase synchronization in both networks when noise strength was increased independently of the Poisson rate ratio p. Again, the beneficial effect of noise was bounded and noise strengths above 7 led to low synchrony within and across networks (Figure 4A).

Furthermore, the results of the one-dimensional exploration of scenario 3 confirmed an inverse relationship between inter-network synchronization measures and within-network synchronization measures over a wide range of noise strengths (Figure 4B), matching the results from scenario 1 . Together with the mean phase coherence the dominant frequency ratio reached high values at a certain strength threshold (at approx. $\sigma^{2}=$ 3.7), while the Kuramoto order parameter of each population decreased nearly monotonically with increasing noise strength.

Again, depending on the Poisson rate ratio, increasing the noise beyond a certain threshold had a detrimental effect on all synchronization measures and eventually led to fluctuating dominant frequency ratios (Figure 4B).

\subsubsection{Analysis of Three Distinct States}

We again investigated the same three different states as for the ING networks (see previous section):

State 1: Unsynchronized activity across and synchronized activity within networks-Weak noise and weak internetwork coupling (Figure 5A)

State 2: Synchronized activity across and within networksWeak noise and strong inter-network coupling (Figure 5B)

State 3: Synchronized activity across and unsynchronized activity within networks-Strong noise and weak internetwork coupling (Figure 5C).

Overall, for all three states we saw clear PING rhythms in both EI networks, where excitatory neurons fired first driving the inhibitory population which subsequently silenced the excitatory activity. After the decay of the inhibition the excitatory neurons fired again and the gamma cycle started over. However, the participation of neurons in each gamma cycle varied for the three states. While it was high for states 1 and 2, it was lower for the third state with strong noise and weak inter-network coupling.

In state 1 (Figure 5A), the low inter-network coupling combined with the weak noise input was not sufficient to synchronize the two networks. Therefore, the dominant frequencies of both networks were solely determined by the Poisson rate ratio $p=0.85$, resulting in a faster rhythm at 68 $\mathrm{Hz}$ for network 1 (which received stronger noise) and a slower rhythm at $58 \mathrm{~Hz}$ for network 2. As the rhythms between the two networks were not synchronized, the phases of the networks showed, unsurprisingly, no coherence.

Once we increased the inter-network coupling strength sufficiently in state 2 , the networks synchronized their rhythms (Figure 5B).

This was expressed by a match of the dominant frequencies at $60 \mathrm{~Hz}$. Noteworthy, the increased coupling slowed down the rhythm of network 1 . The phase plot showed a phase locked state where the phases display a constant difference with each other. Both networks displayed a strong PING rhythm and the increased coupling between the networks, compared to state 1 , reduced the variability in the spike times of the I populations.

In state 3 (Figure 5C), while an increase in the noise strength $\sigma^{2}$ transitioned the networks to a state in which their oscillations were frequency and phase locked, similar to an increase in the inter-network coupling strengths, the synchronization within these networks was considerably decreased which was shown by the high spike variability in the spike raster plots.

Interestingly, in contrast to state 2 where the rhythm of network 1 slowed down to the pace of network 2 , we observed the opposite behavior in state 3 . The slower network 2 sped up and both networks shared a peak activity at $68-70 \mathrm{~Hz}$. Compared to state 1 , in state 3 phase synchronization was increased, although it did not reach the nearly perfect synchronization achieved in state 2 .

Although the PING rhythm was still present in both networks, variability in the firing of inhibitory, as well as excitatory cells was markedly increased by the strong uncorrelated noise. Especially, the firing of the E cells was spread widely across the time interval 
A Within Phase Synchronization - Net 1 - Excitatory

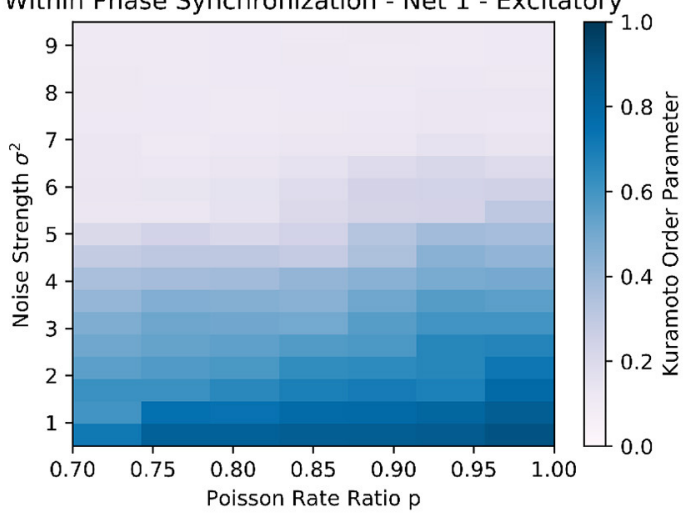

Within Phase Synchronization - Net 2 - Excitatory

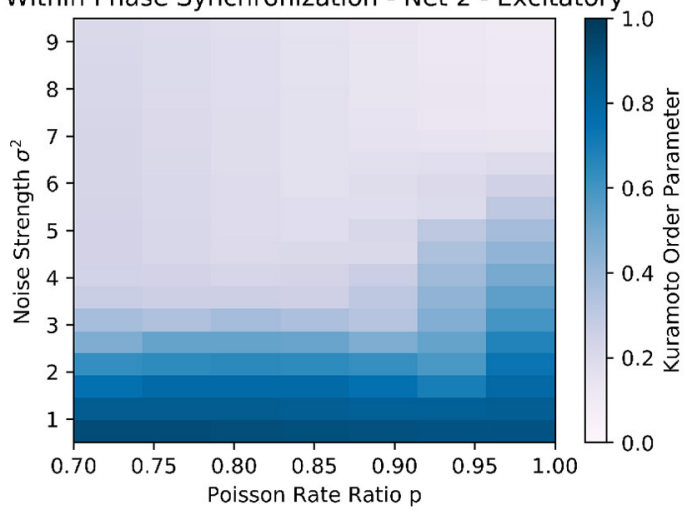

Mean Phase Coherence between Networks

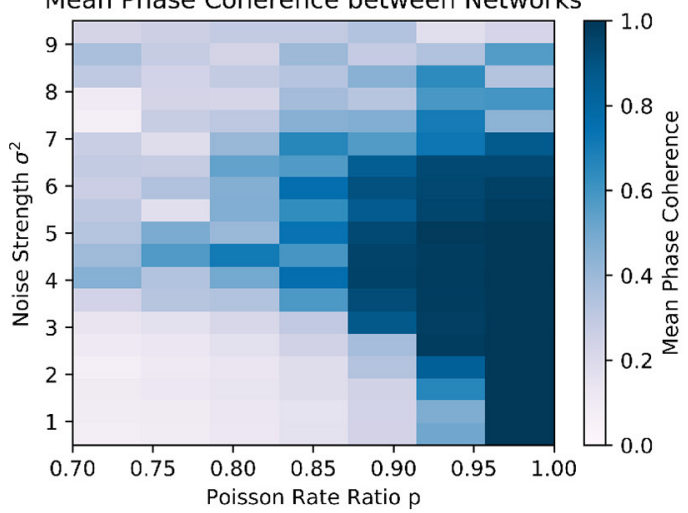

Within Phase Synchronization - Net 1 - Inhibitory

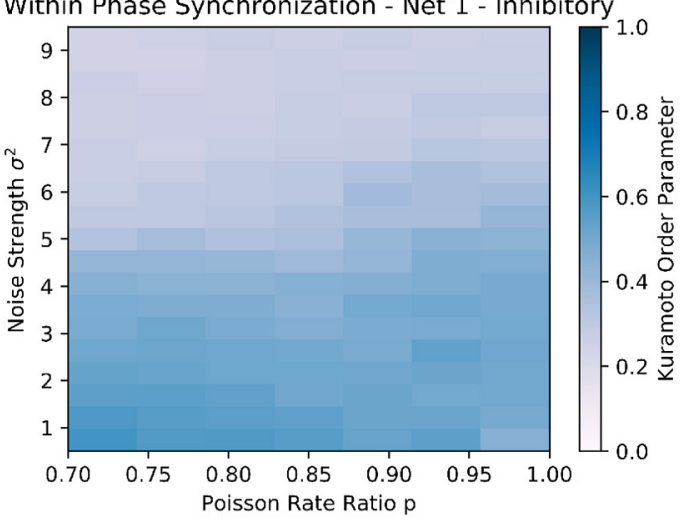

Within Phase Synchronization - Net 2 - Inhibitory
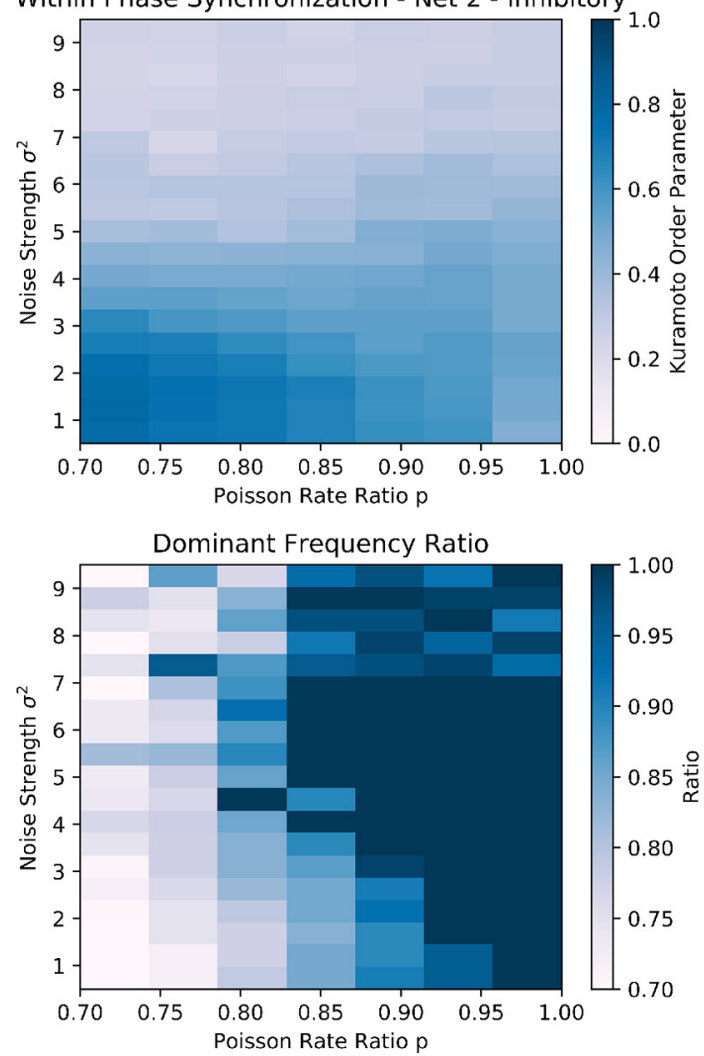

B

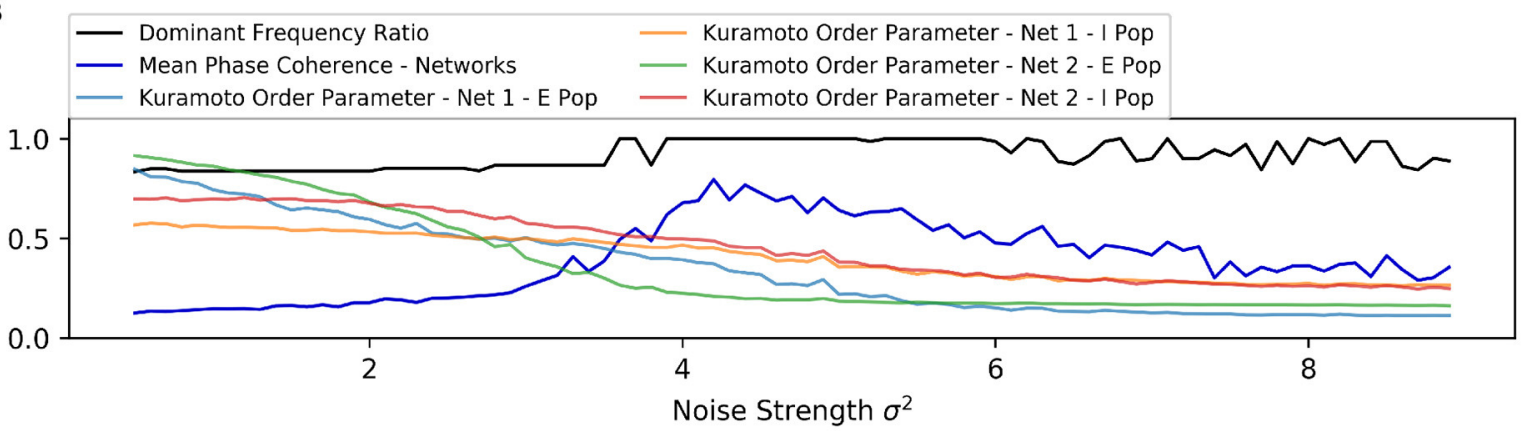

FIGURE 4 | Scenario 3: Exploration of two random sparsely connected excitatory-inhibitory networks driven by the PING mechanism. (A) Exploring the within and across network synchronization behavior over different noise strengths $\sigma^{2}$ and Poisson rate ratio $p$ values. (B) One-dimensional explorations over noise strength $\sigma^{2}$. Poisson rate ratio stayed constant with $p=0.85$. Range of 0.5 to 9.0 in 0.1 steps with runtime of $3 \mathrm{~s}$ for each trial. 


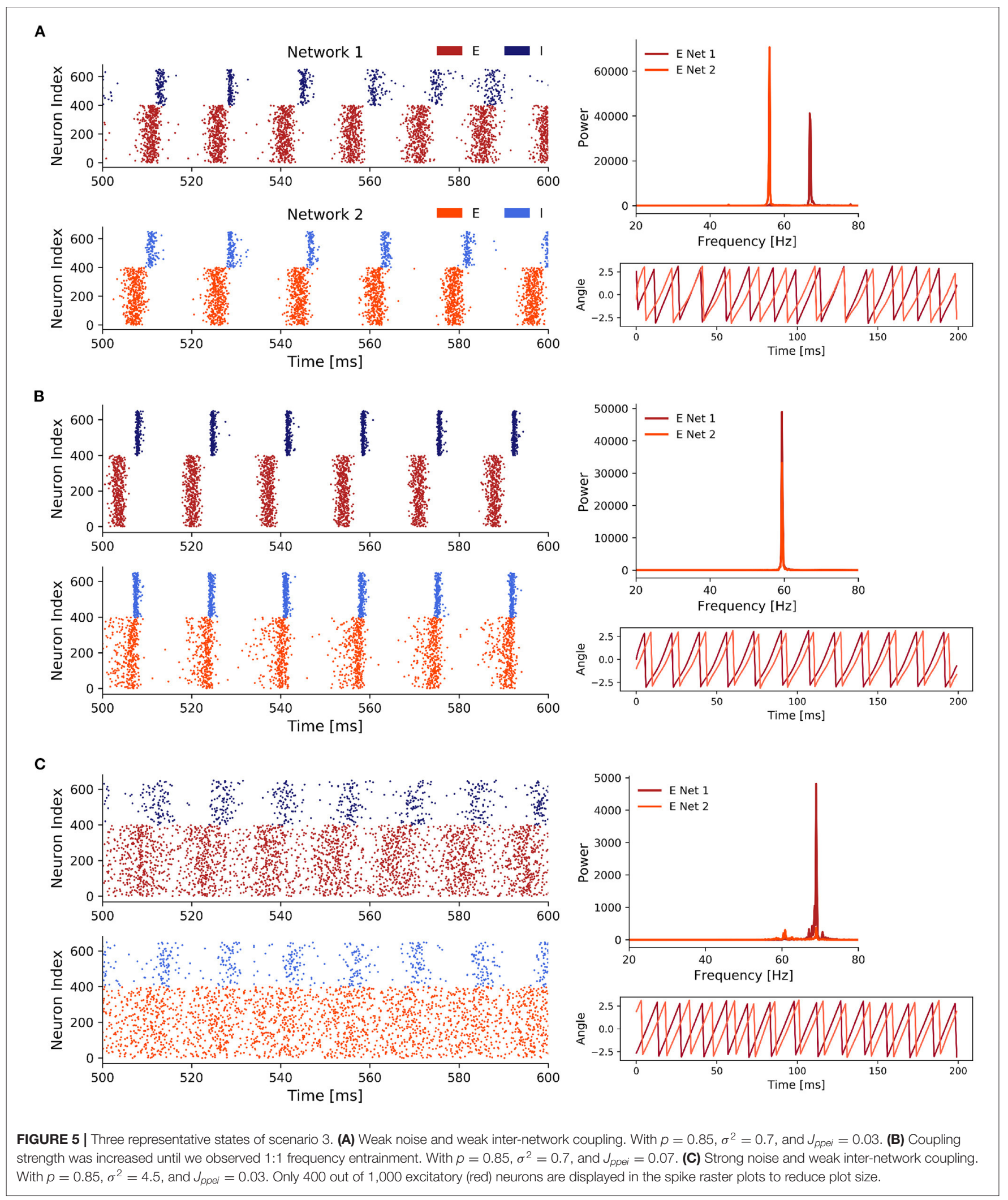


between two consecutive inhibitory firing cycles. This explained the distinct decrease of the power in the frequency spectra compared to state 1 and 2 .

Since LFP proxies based synaptic currents of excitatory cells usually more closely match experimentally recorded LFPs (Mazzoni et al., 2008, 2015; Barbieri et al., 2014), we repeated the analysis of the three distinct states for a current-based LFP proxy. Specifically, we used the sum of the absolute values of AMPA and GABA currents, which captures approximately $90 \%$ of the variance of experimentally measured LFPs (Mazzoni et al., 2015) and did not find any differences. The results are summarized in Supplementary Figure S2.

\subsection{Details of the Synchronization Mechanism}

After demonstrating that strong, uncorrelated was also able to synchronize interconnected EI networks, we looked at the synchronization mechanism in more detail. Again, the mechanism is general to the all-to-all (scenario 2) and sparse random connectivity (scenario 3 ), so we limited our analysis to the latter to minimize redundancy. Based on Meng and Riecke (2018) and our previous analysis of two interacting inhibitory networks (scenario 1), we expected that the increased heterogeneity in the inhibitory membrane potentials caused by sufficiently strong uncorrelated noise would also be a key factor in the noise-induced synchronization between EI networks.

\subsubsection{Heterogeneity in Inhibitory Membrane Potentials}

As is evident from the previous section, the synchronization induced by strong inter-network coupling in state 2 was fundamentally different from synchronization induced by an increase in the noise strengths present in state 3. Specifically, in state 3 the inhibitory cells of the second network can be divided into two groups based on an arbitrary gamma cycle $c_{i}$ of the inhibitory population in the first network (Figure 6A). The first group fired in cycle $c_{i}$ in response to the firing of the excitatory population in network 1 . However, the neurons in the second group stayed suppressed and skipped this cycle. However, they were more likely to fire in the next cycle $c_{i+1}$. Thus, there was always a fraction of neurons that was likely to fire at a cycle $c_{i}$ in response to excitation from network 1 and a remaining fraction that already fired in one of the previous cycles $c_{i-1}, c_{i-2}, \ldots$ and, therefore, skipped cycle $c_{i}$, in order to fire with increased probability in one of the upcoming cycles $c_{i+1}, c_{i+2}, \ldots$.

Overall, the noise-induced variability of the membrane potentials weakened the PING rhythm within the networks and thereby enhanced the responsiveness of neurons to excitation from the respective other network outside of the temporal window of the neurons' network rhythm. This caused the slower network 2 to speed up to the pace of the faster network 1 .

\subsubsection{Inter-spike Interval Histograms}

In order to quantify the observed spike variability, we next took a look the inter-spike interval (ISI) histograms for each population. To get insight into how stronger noise transitions the weak synchrony of state 1 into the high synchrony state 3 , we examined the ISI histograms of the I and $\mathrm{E}$ cells of both networks (Figures 6B,C). For state 1, we observed relatively low variance in all four groups. Importantly, with strongly increased noise the model transitioned from state 1 to state 3 and the variance of the ISIs of both the E and, even more pronounced, the I populations was noticeably increased in state 3 (Figure 6C). Besides an increase in the variance, the mean ISIs $\mu$ increased in both I populations as well. As the dominant frequency in network 1 did not change from state 1 to state 3 and as the dominant frequency in network 2 even increased in state 3 , the higher mean ISI implied a decrease in the participation of I cells in their respective population rhythm.

Interestingly, the ISIs of E cells did not change as much as those of the I cells over the transition from state 1 to state 3, although the $\mathrm{E}$ populations received a higher amount of noise as input (we increased $\sigma^{2}$ from 0.7 to 4.5 ). Conclusively, the increase in the ISI variability and mean of the I populations confirmed the current notion that the noiseinduced heterogeneity of the I cells is a key factor in enhancing synchronization between EI networks.

\section{DISCUSSION}

We explored the role of uncorrelated noise in the synchronization of interacting gamma rhythms. We confirmed prior results from Meng and Riecke (2018) on ING rhythms and extended their findings to gamma oscillations produced by the PING mechanism. To this end, we modeled two interconnected excitatory-inhibitory networks in various network settings and analyzed how synchronization within and across the networks changed depending on the noise strength.

\subsection{Uncorrelated Noise Facilitates Inter-network Synchronization in PING Networks}

We found an optimal range of noise strengths that enhanced across network synchronization. However, the beneficial effect of noise was limited and we detected an upper and lower bound in all three scenarios. Noise strengths above the upper bound eventually broke the network rhythms as spiking of neurons was mainly determined by the uncorrelated input, indicated by a worsening of within and across network phase synchronization. On the other hand, noise strengths below the lower bound were not able to dampen the within network coupling sufficiently to enhance the responsiveness of neurons to input from the respective other network.

\subsection{Noise-Induced Synchronization Mechanism}

Further, we confirmed that the mechanism described by Meng and Riecke (2018) that underlies the noise-induced synchronization across networks in inhibitory networks was also present in the PING networks considered here. Although the two investigated variants of two interacting EI networks differed in several points from inhibitory networks, the same fundamental mechanism could be observed. Noise caused considerable voltage 
A
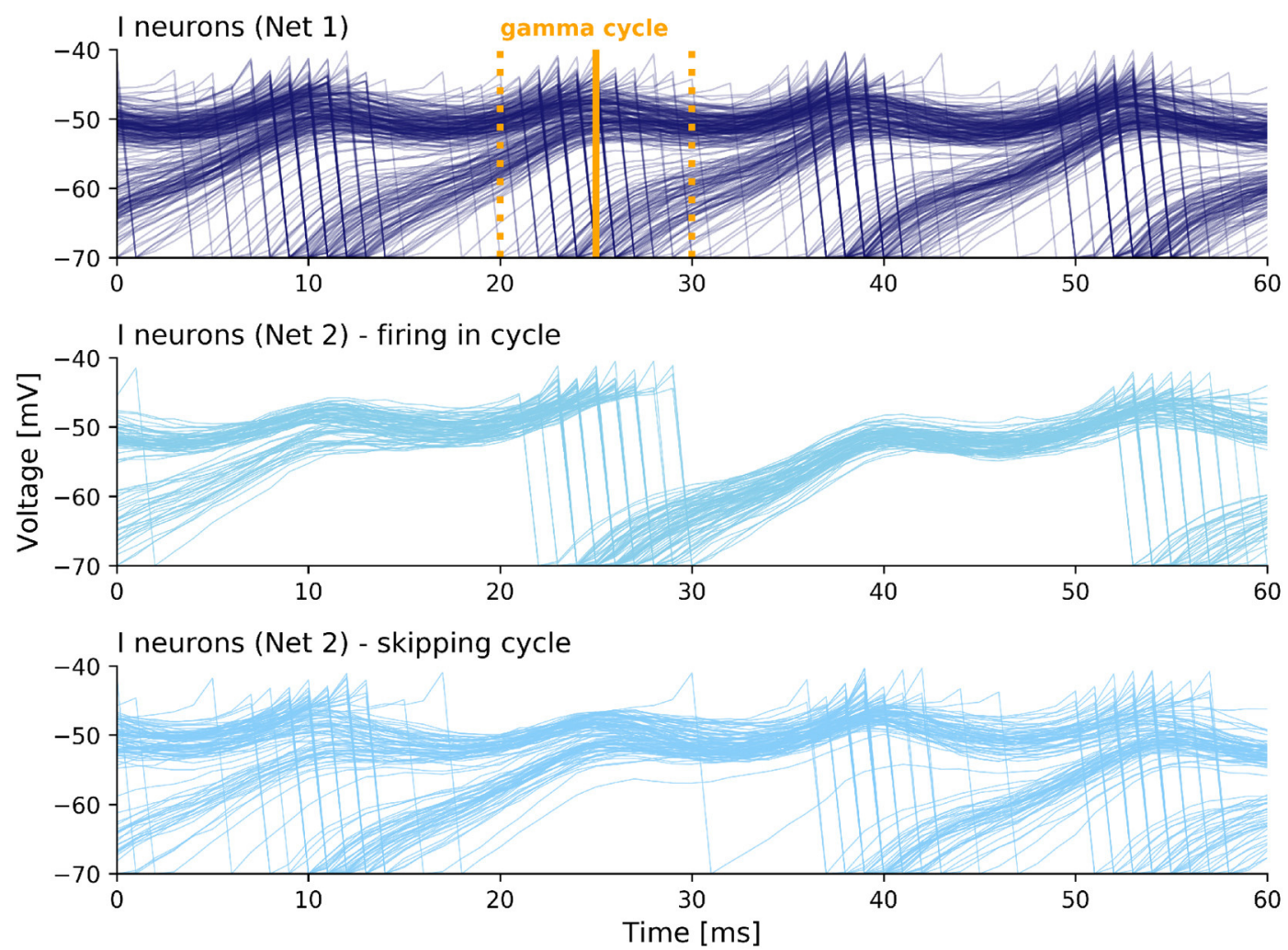

B
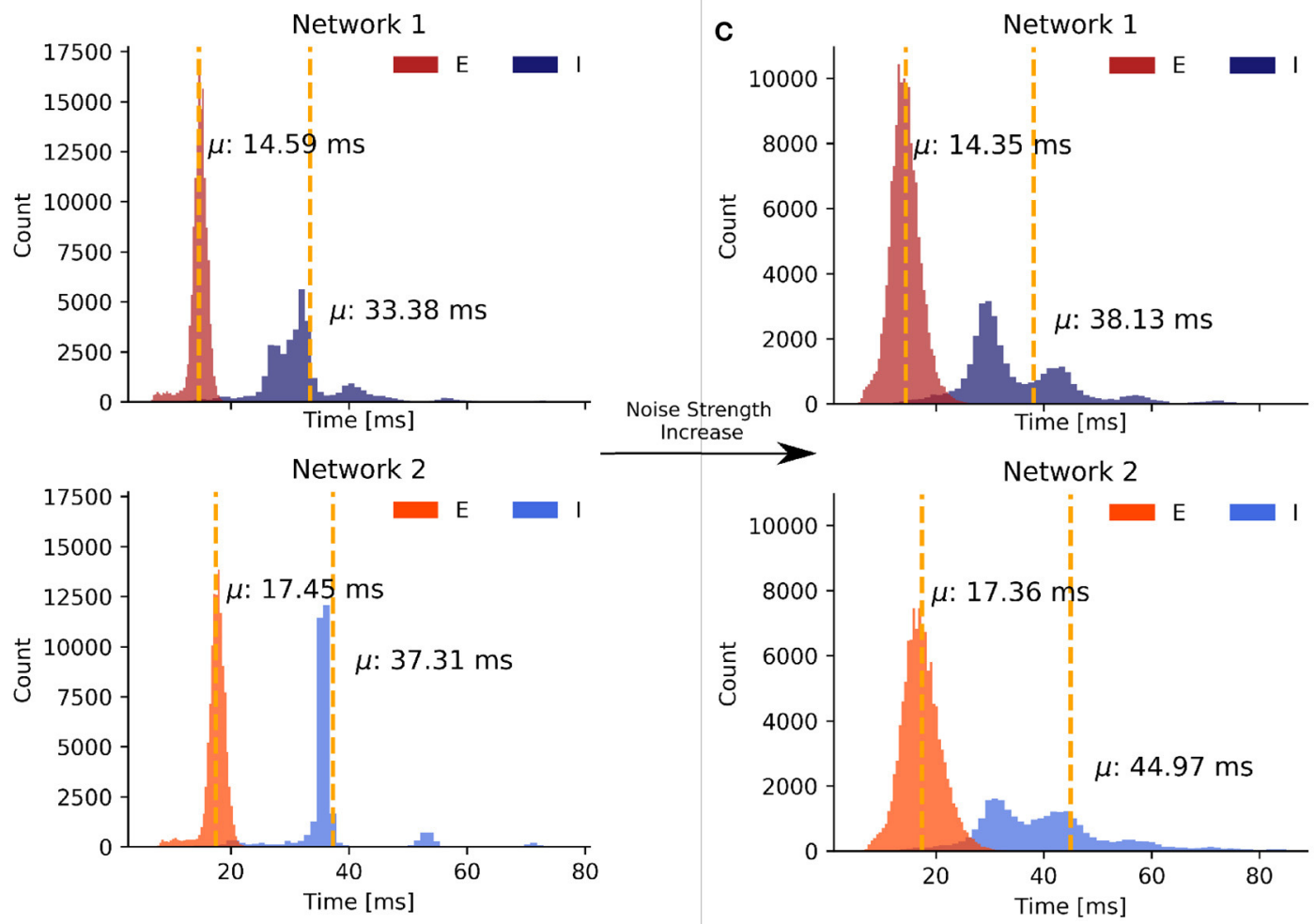

FIGURE 6 | Synchronization mechanism based on uncorrelated noise in scenario 3. (A) The first plot displays voltage traces of I neurons in network 1 while the second and third plot display grouped voltage traces of I neurons in network 2 . We selected an arbitrary I cycle $c_{j}$ of network 1 in state 3 marked by the time window $\left[t_{\text {start }}, t_{\text {end }}\right]$ (yellow lines). A fraction of neurons participated in the selected cycle (second plot) while the remaining neurons skipped the cycle and sparsely participated in the previous and next cycle (third plot). The values on the $\mathrm{x}$ axis are relative. (B) ISI histogram of state 1 with weak coupling and weak noise. (C) ISI histogram of state 3 with weak coupling and strong noise. 
fluctuations in all neurons, thereby weakening the PING rhythm within a network. This led to an increased responsiveness of neurons in one network to excitation from the respective other network as a fraction of neurons was likely to be close to its membrane threshold. This enabled a variable fraction of neurons to spike in response to excitation from the other network, thereby promoting the network rhythms to synchronize over time with the activity of the faster network. Noteworthy, in the case of inhibitory networks, it was not excitation of the faster network that sped up the rhythm of the slower network. Instead, inhibition of the faster network gated the slower network similar to the ING mechanism itself (Meng and Riecke, 2018).

Importantly, the identified synchronization mechanism is fundamentally different from stochastic synchronization promoted by correlations in the external noise input (as already discussed in Meng and Riecke, 2018). Further, it also differs from stochastic resonance (Longtin et al., 1991; Douglass et al., 1993) or the related phenomenon of enhanced responsiveness (Destexhe and Rudolph-Lilith, 2012) where noise amplifies a weak input stimulus so that it can be detected by the neuron. While the identified mechanism enhanced the responsiveness of neurons to excitation from the other network, it did not amplify a weak signal but instead weakened the rhythm within a network. This rendered neurons susceptible to spikes in response to excitation or inhibition outside of the temporal window defined by the networks own rhythm. We also showed that the noise-induced synchronization is different from synchronization introduced through strong coupling between the networks and that they show different signatures. While coupling-induced synchronization leads to a high within- and between-network synchronization, noise-induced synchronization shows weaker within-network synchronization together with high between-network synchronization. Furthermore, noise-induced synchronization led to a speed up of the slower network to match the oscillation frequency of the faster network, whereas synchronization through strong coupling resulted in a slowing down of the fast network. We propose the following view: the fundamental aspect of the noise-induced synchronization mechanism is the desynchronization within a network that enhances the responsiveness of neurons to any external stimulation outside of the short temporal window defined by the ING or PING mechanism, in this case a second network with a faster rhythm.

As the spiking behavior of the $\mathrm{E}$ population did not considerably vary across scenarios, we hypothesize that noiseinduced variability in the inhibitory population is central to enhancing synchronization between networks and that the desynchronization of the $\mathrm{E}$ population has a supporting role by facilitating the heterogeneity of the inhibitory population.

\subsection{Role of Uncorrelated Noise in Aberrant Neuronal Communication}

Importantly, the communication through coherence (CTC) hypothesis suggests that neuronal communication among neuronal groups is mediated by phase synchronization (Fries, 2005, 2015). Conclusively, our findings suggest that uncorrelated noise, mimicking the strong synaptic noise observed in the cerebral cortex (Destexhe et al., 2003), can have a supporting role in facilitating neuronal communication among neuronal networks displaying rhythmic gamma band activity. In specific, there exists an optimal level of noise that allows the transition of networks from a desynchronized state to a synchronized state by enhancing the responsiveness of neurons inside a network to inhibitory and excitatory input originating from another network. This suggests that deficits in sensory or cognitive abilities as seen in several neurological or psychiatric disorders which have been hypothesized to be related to aberrant synchronization in the gamma band, might be due to an increase in the signal-to-noise ratio that is often seen in these disorders. For example, patients with schizophrenia show deficits in visual Gestalt perception (Spencer and Ghorashi, 2014) and working memory performance (Chen et al., 2014), two cognitive processes that have been linked to gamma oscillations. Furthermore, it has been shown that the signal-to-noise ratio in neural activity of patients with schizophrenia is significantly decreased (Winterer et al., 2000, 2004, 2006; Rolls et al., 2008). Therefore, some of these deficits might be attributable to a decreased ability of inter-network synchronizability in the gamma band due to the increased noise levels, which push the network of the range of noise strengths to a point where the noise is so strong that it hinders inter-regional synchronization. Another situation might be that the increased noise strength actually pushes the network further within the parameter range that facilitates synchronization, resulting, however, in enhanced synchronization between region which should not be strongly synchronized, i.e., generating a hypersynchronized state. Both possibilities could be distinguished by their differential effects on functional connectivity (FC) (if measured via synchronization). The first possibility would lead to an overall decrease in FC, whereas the second would lead to an increase of FC.

\subsection{Limitations}

The model we employed in our explorations is of course a highly simplified representation of neural populations in vivo. For instance, our model currently represents a standard EI model of one excitatory and one inhibitory population and ignores the existence of multiple inhibitory populations of different interneuron types. We only modeled fast-spiking parvalbumin-expressing $\left(\mathrm{PV}^{+}\right)$interneurons since their somatic inhibition of regulates the timing of action potentials and imposes brief time windows in which PCs can spike, therefore, promoting synchronization (Silberberg, 2008). Furthermore, $\mathrm{PV}^{+}$interneurons seem to be responsible for the generation of gamma oscillations (Hájos et al., 2004; Bartos et al., 2007; Cardin et al., 2009). However, somatostatin-expressing $\left(\mathrm{SST}^{+}\right)$and vasoactive peptide-expressing $\left(\mathrm{VIP}^{+}\right)$interneurons contribute substantially to regulating GABAergic inhibition in the cortex (Rossignol, 2011; Pfeffer et al., 2013; Veit et al., 2017) and dysfunction of these interneurons are associated with psychiatric disorders (Rossignol, 2011; Fung et al., 2014). Additionally, $\mathrm{SST}^{+}$interneurons are presumed to play an 
important role in synchronization of visually induced, contextdependent gamma rhythms in visual cortex (Veit et al., 2017).

Additionally, our model does not represent the layered structure of cortex and therefore cannot capture the intricate differences in information processing between different layers of cortical regions and their oscillatory signatures.

Furthermore, in our current model we simply feed external, uncorrelated noise into the two networks and do not model the origin of this noise. Further studies are warranted to elucidate the effect of different sources of uncorrelated noise in cortical networks, such as stochastic synaptic transmission and ion-channel noise (Faisal et al., 2008; Renart and Machens, 2014), on the synchronization mechanism described here. We also did not study the interaction this noise-induced synchronization mechanism with other synchronization mechanisms due to correlated noise, such as stochastic synchronization (Pikovskii, 1984; Mainen and Sejnowski, 1995; Shea-Brown et al., 2008; Abouzeid and Ermentrout, 2011), that have been described before.

\section{DATA AVAILABILITY STATEMENT}

The original contributions presented in the study are included in the article/Supplementary Material, further inquiries can be directed to the corresponding author/s.

\section{REFERENCES}

Abouzeid, A., and Ermentrout, B. (2011). Correlation transfer in stochastically driven neural oscillators over long and short time scales. Phys. Rev. E 84:061914. doi: 10.1103/PhysRevE.84.061914

Barbieri, F., Mazzoni, A., Logothetis, N. K., Panzeri, S., and Brunel, N. (2014). Stimulus dependence of local field potential spectra: experiment versus theory. J. Neurosci. 34, 14589-14605. doi: 10.1523/JNEUROSCI.5365-13.2014

Bartos, M., Vida, I., and Jonas, P. (2007). Synaptic mechanisms of synchronized gamma oscillations in inhibitory interneuron networks. Nat. Rev. Neurosci. 8, 45-56. doi: 10.1038/nrn2044

Besserve, M., Lowe, S. C., Logothetis, N. K., Schölkopf, B., and Panzeri, S. (2015). Shifts of gamma phase across primary visual cortical sites reflect dynamic stimulus-modulated information transfer. PLoS Biol. 13:e1002257. doi: 10.1371/journal.pbio.1002257

Börgers, C., Epstein, S., and Kopell, N. J. (2005). Background gamma rhythmicity and attention in cortical local circuits: a computational study. Proc. Natl Acad. Sci. 102, 7002-7007. doi: 10.1073/pnas.0502366102

Börgers, C. and Kopell, N. (2003). Synchronization in networks of excitatory and inhibitory neurons with sparse, random connectivity. Neural Comput. 15, 509-538. doi: 10.1162/089976603321192059

Bosman, C. A., Schoffelen, J.-M., Brunet, N., Oostenveld, R., Bastos, A. M., Womelsdorf, T., et al. (2012). Attentional stimulus selection through selective synchronization between monkey visual areas. Neuron $75,875-888$. doi: 10.1016/j.neuron.2012.06.037

Brette, R., and Gerstner, W. (2005). Adaptive exponential integrate-and-fire model as an effective description of neuronal activity. J. Neurophysiol. 94, 3637-3642. doi: 10.1152/jn.00686.2005

Buzsáki, G. (2006). Rhythms of the Brain. Oxford: Oxford University Press.

Buzsáki, G., and Draguhn, A. (2004). Neuronal oscillations in cortical networks. Science 304, 1926-1929. doi: 10.1126/science.1099745

\section{AUTHOR CONTRIBUTIONS}

LR and CM designed the study and wrote the initial draft of the manuscript. LR implemented the models and ran the experiments and performed the analyses. CM supervised the implementation, the running of the experiments and the analyses. LR, KO, and CM discussed the findings. All authors contributed to manuscript revision, read, and approved the submitted version.

\section{FUNDING}

We acknowledge support by the German Research Foundation and the Open Access Publication Fund of TU Berlin.

\section{ACKNOWLEDGMENTS}

We would like to thank J. H. Meng and H. Riecke for providing their code to generate the noise input from their original study and for answering questions about implementation details of their model.

\section{SUPPLEMENTARY MATERIAL}

The Supplementary Material for this article can be found online at: https://www.frontiersin.org/articles/10.3389/fncom. 2022.825865/full\#supplementary-material

Buzsáki, G., and Wang, X.-J. (2012). Mechanisms of gamma oscillations. Ann. Rev. Neurosci. 35, 203-225. doi: 10.1146/annurev-neuro-062111-150444

Cakan, C., and Rebscher, L. (2020). Caglorithm/Mopet: Release With doi. Zenodo. Asvailable online at: https://doi.org/10.5281/zenodo.3941539

Cardin, J. A., Carlén, M., Meletis, K., Knoblich, U., Zhang, F., Deisseroth, K., et al. (2009). Driving fast-spiking cells induces gamma rhythm and controls sensory responses. Nature 459, 663-667. doi: 10.1038/nature 08002

Chen, C.-M. A., Stanford, A. D., Mao, X., Abi-Dargham, A., Shungu, D. C., Lisanby, S. H., et al. (2014). Gaba level, gamma oscillation, and working memory performance in schizophrenia. NeuroImage Clin. 4, 531-539. doi: 10.1016/j.nicl.2014.03.007

Destexhe, A., Rudolph, M., and Paré, D. (2003). The high-conductance state of neocortical neurons in vivo. Nat. Rev. Neurosci. 4, 739-751. doi: $10.1038 / \mathrm{nrn} 1198$

Destexhe, A., and Rudolph-Lilith, M. (2012). Neuronal Noise, Vol 8 (Berlin: Springer Science \& Business Media).

Douglass, J. K., Wilkens, L., Pantazelou, E., and Moss, F. (1993). Noise enhancement of information transfer in crayfish mechanoreceptors by stochastic resonance. Nature 365, 337-340. doi: 10.1038/365337a0

Engel, A. K., Fries, P., and Singer, W. (2001). Dynamic predictions: oscillations and synchrony in top-down processing. Nat. Rev. Neurosci. 2, 704-716. doi: $10.1038 / 35094565$

Fabri, M., and Manzoni, T. (1996). Glutamate decar ylase immunoreactivity in corticocortical projecting neurons of rat somatic sensory cortex. Neuroscience 72, 435-448. doi: 10.1016/0306-4522(95)00568-4

Fabri, M., and Manzoni, T. (2004). Glutamic acid decarboxylase immunoreactivity in callosal projecting neurons of cat and rat somatic sensory areas. Neuroscience 123, 557-566. doi: 10.1016/j.neuroscience.2003.09.011

Faisal, A. A., Selen, L. P., and Wolpert, D. M. (2008). Noise in the nervous system. Nat. Rev. Neurosci. 9, 292-303. doi: 10.1038/nrn2258 
Fries, P. (2005). A mechanism for cognitive dynamics: neuronal communication through neuronal coherence. Trends Cogn. Sci. 9, 474-480. doi: $10.1016 /$ j.tics.2005.08.011

Fries, P. (2009). Neuronal gamma-band synchronization as a fundamental process in cortical computation. Ann. Rev. Neurosci. 32, 209-224. doi: 10.1146/annurev.neuro.051508.135603

Fries, P. (2015). Rhythms for cognition: communication through coherence. Neuron 88, 220-235. doi: 10.1016/j.neuron.2015.09.034

Fries, P., Reynolds, J. H., Rorie, A. E., and Desimone, R. (2001). Modulation of oscillatory neuronal synchronization by selective visual attention. Science 291, 1560-1563. doi: 10.1126/science.1055465

Fung, S. J., Fillman, S. G., Webster, M. J., and Weickert, C. S. (2014). Schizophrenia and bipolar disorder show both common and distinct changes in cortical interneuron markers. Schizophrenia Res. 155, 26-30. doi: 10.1016/j.schres.2014.02.021

Gluckman, B. J., Netoff, T. I., Neel, E. J., Ditto, W. L., Spano, M. L., and Schiff, S. J. (1996). Stochastic resonance in a neuronal network from mammalian brain. Phys. Rev. Lett. 77:4098. doi: 10.1103/PhysRevLett.77.4098

Goldobin, D. S., and Pikovsky, A. (2005). Synchronization and desynchronization of self-sustained oscillators by common noise. Phys. Revi. E 71:045201. doi: 10.1103/PhysRevE.71.045201

Gonchar, Y., Johnson, P., and Weinberg, R. (1995). Gaba-immunopositive neurons in rat neocortex with contralateral projections to si. Brain Res. 697, 27-34. doi: 10.1016/0006-8993(95)00746-D

Gray, C. M., König, P., Engel, A. K., and Singer, W. (1989). Oscillatory responses in cat visual cortex exhibit inter-columnar synchronization which reflects global stimulus properties. Nature 338, 334-337. doi: 10.1038/338334a0

Grothe, I., Neitzel, S. D., Mandon, S., and Kreiter, A. K. (2012). Switching neuronal inputs by differential modulations of gamma-band phase-coherence. J. Neurosci. 32, 16172-16180. doi: 10.1523/JNEUROSCI.0890-12.2012

Hájos, N., Pálhalmi, J., Mann, E. O., Németh, B., Paulsen, O., and Freund, T. F. (2004). Spike timing of distinct types of gabaergic interneuron during hippocampal gamma oscillations in vitro. J. Neurosci. 24, 9127-9137. doi: 10.1523/JNEUROSCI.2113-04.2004

Hänggi, P. (2002). Stochastic resonance in biology: how noise can enhance detection of weak signals and help improve biological information processing. ChemPhysChem 3, 285-290. doi: 10.1002/1439-7641(20020315)3:3<285::AID-CPHC285>3.0.CO;2-A

Longtin, A., Bulsara, A., and Moss, F. (1991). Time-interval sequences in bistable systems and the noise-induced transmission of information by sensory neurons. Phys. Rev. Lett. 67, 656. doi: 10.1103/PhysRevLett.67.656

Mainen, Z. F., and Sejnowski, T. J. (1995). Reliability of spike timing in neocortical neurons. Science 268, 1503-1506. doi: 10.1126/science.7770778

Mazzoni, A., Lindén, H., Cuntz, H., Lansner, A., Panzeri, S., and Einevoll, G. T. (2015). Computing the local field potential (lfp) from integrate-and-fire network models. PLoS Comput. Biol. 11:e1004584. doi: 10.1371/journal.pcbi.1004584

Mazzoni, A., Panzeri, S., Logothetis, N. K., and Brunel, N. (2008). Encoding of naturalistic stimuli by local field potential spectra in networks of excitatory and inhibitory neurons. PLoS Comput. Biol. 4:e1000239. doi: 10.1371/journal.pcbi.1000239

McDonald, C., and Burkhalter, A. (1993). Organization of long-range inhibitory connections within rat visual cortex. J. Neurosci. 13, 35-62. doi: 10.1523/JNEUROSCI.13-02-00768.1993

McDonnell, M. D., and Ward, L. M. (2011). The benefits of noise in neural systems: bridging theory and experiment. Nat. Rev. Neurosci. 12, 415-425. doi: $10.1038 / \mathrm{nrn} 3061$

McMillen, D., and Kopell, N. (2003). Noise-stabilized long-distance synchronization in populations of model neurons. J. Comput. Neurosci. 15, 143-157. doi: 10.1023/A:1025860724292

Melloni, L., Molina, C., Pena, M., Torres, D., Singer, W., and Rodriguez, E. (2007). Synchronization of neural activity across cortical areas correlates with conscious perception. J. Neurosci. 27, 2858-2865. doi: 10.1523/JNEUROSCI.4623-06.2007

Meng, J. H., and Riecke, H. (2018). Synchronization by uncorrelated noise: interacting rhythms in interconnected oscillator networks. Sci. Rep. 8:6949. doi: 10.1038/s41598-018-24670-y
Ni, J., Wunderle, T., Lewis, C. M., Desimone, R., Diester, I., and Fries, P. (2016). Gamma-rhythmic gain modulation. Neuron 92, 240-251. doi: 10.1016/j.neuron.2016.09.003

Pfeffer, C. K., Xue, M., He, M., Huang, Z. J., and Scanziani, M. (2013). Inhibition of inhibition in visual cortex: the logic of connections between molecularly distinct interneurons. Nat. Neurosci. 16, 1068-1076. doi: 10.1038/nn.3446

Pikovskii, A. (1984). Synchronization and stochastization of nonlinear oscillations by external noise. Nonlin. Turbulent Processes Phys. 2:1601.

Pikovsky, A., Rosenblum, M., and Kurths, J. (2003). Synchronization: a Universal Concept in Nonlinear Sciences. Cambridge: Cambridge University Press.

Plesser, H. E., and Tanaka, S. (1997). Stochastic resonance in a model neuron with reset. Phys. Lett. A 225, 228-234. doi: 10.1016/S0375-9601(96)00878-X

Renart, A. and Machens, C. K. (2014). Variability in neural activity and behavior. Curr. Opin. Neurobiol. 25, 211-220. doi: 10.1016/j.conb.2014.02.013

Rolls, E. T., Loh, M., Deco, G., and Winterer, G. (2008). Computational models of schizophrenia and dopamine modulation in the prefrontal cortex. Nat. Rev. Neurosci. 9, 696-709. doi: 10.1038/nrn2462

Rossignol, E. (2011). Genetics and function of neocortical gabaergic interneurons in neurodevelopmental disorders. Neural Plasticity 2011:649325. doi: $10.1155 / 2011 / 649325$

Shea-Brown, E., Josić, K., De La Rocha, J., and Doiron, B. (2008). Correlation and synchrony transfer in integrate-and-fire neurons: Basic properties and consequences for coding. Phys. Rev. Lett. 100:108102. doi: 10.1103/PhysRevLett.100.108102

Silberberg, G. (2008). Polysynaptic subcircuits in the neocortex: spatial and temporal diversity. Curr. Opin. Neurobiol. 18, 332-337. doi: 10.1016/j.conb.2008.08.009

Singer, W. (1999). Neuronal synchrony: a versatile code for the definition of relations? Neuron 24, 49-65. doi: 10.1016/S0896-6273(00)80821-1

Spencer, K. M. and Ghorashi, S. (2014). Oscillatory dynamics of gestalt perception in schizophrenia revisited. Front. Psychol. 5:68. doi: 10.3389/fpsyg.2014.00068

Stimberg, M., Brette, R., and Goodman, D. F. (2019). Brian 2, an intuitive and efficient neural simulator. eLife 8:e47314. doi: 10.7554/eLife.47314

Tallon-Baudry, C., Bertrand, O., Peronnet, F., and Pernier, J. (1998). Induced $\gamma$ band activity during the delay of a visual short-term memory task in humans. J. Neurosci. 18, 4244-4254. doi: 10.1523/JNEUROSCI.18-11-04244.1998

Tiesinga, P. and Sejnowski, T. J. (2009). Cortical enlightenment: are attentional gamma oscillations driven by ing or ping? Neuron $63,727-732$. doi: 10.1016/j.neuron.2009.09.009

Tomioka, R. and Rockland, K. S. (2007). Long-distance corticocortical gabaergic neurons in the adult monkey white and gray matter. J. Comparative Neurol. 505, 526-538. doi: 10.1002/cne.21504

Uhlhaas, P. J., and Singer, W. (2006). Neural synchrony in brain disorders: relevance for cognitive dysfunctions and pathophysiology. Neuron 52, 155-168. doi: 10.1016/j.neuron.2006.09.020

Uhlhaas, P. J., and Singer, W. (2010). Abnormal neural oscillations and synchrony in schizophrenia. Nat. Rev. Neurosci. 11, 100-113. doi: 10.1038/nrn2774

Varela, F., Lachaux, J.-P., Rodriguez, E., and Martinerie, J. (2001). The brainweb: phase synchronization and large-scale integration. Nat. Re. Neurosci. 2, 229239. doi: $10.1038 / 35067550$

Veit, J., Hakim, R., Jadi, M. P., Sejnowski, T. J., and Adesnik, H. (2017). Cortical gamma band synchronization through somatostatin interneurons. Nat. Neurosci. 20:951. doi: 10.1038/nn.4562

Ward, L. M., MacLean, S. E., and Kirschner, A. (2010). Stochastic resonance modulates neural synchronization within and between cortical sources. PLoS ONE 5:e14371. doi: 10.1371/journal.pone.0014371

Welch, P. (1967). The use of fast fourier transform for the estimation of power spectra: a method based on time averaging over short, modified periodograms. IEEE Trans. Audio Electroacoust. 15, 70-73. doi: 10.1109/TAU.1967.1161901

Wespatat, V., Tennigkeit, F., and Singer, W. (2004). Phase sensitivity of synaptic modifications in oscillating cells of rat visual cortex. J. Neurosci. 24, 9067-9075. doi: 10.1523/JNEUROSCI.2221-04.2004

Whittington, M. A., Cunningham, M. O., LeBeau, F. E., Racca, C., and Traub, R. D. (2011). Multiple origins of the cortical gamma rhythm. Develop. Neurobiol. 71, 92-106. doi: 10.1002/dneu.20814

Whittington, M. A., Traub, R., Kopell, N., Ermentrout, B., and Buhl, E. (2000). Inhibition-based rhythms: experimental and mathematical 
observations on network dynamics. Int. J. Psychophysiol. 38, 315-336. doi: 10.1016/S0167-8760(00)00173-2

Winterer, G., Coppola, R., Goldberg, T. E., Egan, M. F., Jones, D. W., Sanchez, C. E., et al. (2004). Prefrontal broadband noise, working memory, and genetic risk for schizophrenia. Am. J. Psychiatry 161, 490-500. doi: 10.1176/appi.ajp.161.3.490

Winterer, G., Musso, F., Beckmann, C., Mattay, V., Egan, M. F., Jones, D. W., et al. (2006). Instability of prefrontal signal processing in schizophrenia. Am. J. Psychiatry 163, 1960-1968. doi: 10.1176/ajp.2006.163.11.1960

Winterer, G., Ziller, M., Dorn, H., Frick, K., Mulert, C., Wuebben, Y., et al. (2000). Schizophrenia: reduced signal-to-noise ratio and impaired phaselocking during information processing. Clin. Neurophysiol. 111, 837-849. doi: 10.1016/S0920-9964(00)90663-7

Womelsdorf, T., Schoffelen, J.-M., Oostenveld, R., Singer, W., Desimone, R., Engel, A. K., et al. (2007). Modulation of neuronal interactions through neuronal synchronization. Science 316, 1609-1612. doi: 10.1126/science. 1139597

Zhou, C., Kurths, J., Kiss, I. Z., and Hudson, J. L. (2002). Noise-enhanced phase synchronization of chaotic oscillators. Phys. Rev. Lett. 89:014101. doi: 10.1103/PhysRevLett.89.014101

Zhou, P., Burton, S., Urban, N., and Ermentrout, G. B. (2013). Impact of neuronal heterogeneity on correlated colored noise-induced synchronization. Front. Comput. Neurosci. 7:113. doi: 10.3389/fncom.2013. 00113

Conflict of Interest: The authors declare that the research was conducted in the absence of any commercial or financial relationships that could be construed as a potential conflict of interest.

Publisher's Note: All claims expressed in this article are solely those of the authors and do not necessarily represent those of their affiliated organizations, or those of the publisher, the editors and the reviewers. Any product that may be evaluated in this article, or claim that may be made by its manufacturer, is not guaranteed or endorsed by the publisher.

Copyright (C) 2022 Rebscher, Obermayer and Metzner. This is an open-access article distributed under the terms of the Creative Commons Attribution License (CC BY). The use, distribution or reproduction in other forums is permitted, provided the original author(s) and the copyright owner(s) are credited and that the original publication in this journal is cited, in accordance with accepted academic practice. No use, distribution or reproduction is permitted which does not comply with these terms. 